\title{
Nutrizione iodica globale nel 2020
}

\author{
Antonella Olivieri ${ }^{1} \cdot$ Paolo Vitti $^{2} \cdot$ Francesco Giorgino $^{3} \cdot$ Francesco Trimarchi ${ }^{4}$
}

Accettato: 27 agosto 2020 / Pubblicato online: 10 novembre 2020

(c) Springer Nature Switzerland AG 2020

La Iodine Global Network (IGN) ha recentemente pubblicato lo stato della nutrizione iodica nel mondo nel 2020 [1]. Il documento riporta una nutrizione iodica ottimale in 115 paesi, insufficiente in 23 (inclusi alcuni paesi europei quali Finlandia, Germania e Norvegia) ed eccessiva in 14 (Figg. 1, 2). Lo stesso documento evidenzia come alcuni paesi, precedentemente classificati come iodosufficienti, abbiano progressivamente subito una riduzione dell'apporto alimentare di iodio a livello di popolazione e siano attualmente classificati come iodocarenti (in Europa la Germania), sottoli-

A. Olivieri - in collaborazione con gli Osservatori Regionali per la Prevenzione del Gozzo: Daniela Agrimi (Brindisi, Puglia), Sebastiano Andò, Daniela Bonofiglio (Cosenza, Calabria), Marcello Bagnasco (Genova, Liguria), Pasquale Bellitti (Matera, Basilicata), Francesco Boi (Cagliari, Sardegna), Giulio Doveri (Aosta, Valle D’Aosta), Maurizio Gasperi (Campobasso, Molise), Pietro Lanzetta (Salerno, Campania), Domenico Meringolo (Bologna, Emilia Romagna), Caterina Mian (Padova, Veneto e Friuli Venezia Giulia), Giorgio Napolitano (Chieti, Abruzzo), Fabio Orlandi (Torino, Piemonte), Efisio Puxeddu (Perugia, Umbria), Giorgio Radetti (Bolzano, Trentino Alto Adige), Concetto Regalbuto, Mariacarla Moleti (Catania e Messina, Sicilia), Augusto Taccaliti (Ancona, Marche), Maria Laura Tanda (Varese, Lombardia), Massimo Tonacchera (Pisa, Toscana), Salvatore Ulisse (Roma, Lazio).

\footnotetext{
$\bowtie$ F. Trimarchi

francesco.trimarchi@unime.it

1 Dipartimento di Malattie Cardiovascolari, Endocrino-Metaboliche e Invecchiamento, Istituto Superiore di Sanità, Roma, Italia

2 Dipartimento di Medicina Clinica e Sperimentale, Università di Pisa, Pisa, Italia

3 Sezione di Medicina Interna, Endocrinologia, Andrologia e Malattie Metaboliche, Dipartimento dell'Emergenza e dei Trapianti di Organi, Università degli Studi di Bari Aldo Moro, Bari, Italia

4 Dipartimento di Medicina Clinica e Sperimentale, Università di Messina, Messina, Italia
}

neando la necessità di un continuo monitoraggio dello stato nutrizionale iodico della popolazione.

L'Italia, storicamente iodocarente [2-4], è finalmente entrata a far parte di quei paesi in cui la nutrizione iodica è adeguata e sufficiente a garantire l'equilibrata funzione della ghiandola tiroide, così come dimostrato dalla seconda sorveglianza sulla nutrizione iodica in Italia (periodo 2015-2019) condotta dall'Osservatorio Nazionale per il Monitoraggio della Iodoprofilassi in Italia (OSNAMI) dell'Istituto Superiore di Sanità in collaborazione con gli Osservatori Regionali per la Prevenzione del Gozzo [5]. L'indagine, che è stata realizzata su circa 4.000 bambini in età scolare e residenti in 9 regioni rappresentative del Nord, Centro e Sud Italia, ha dimostrato come nel nostro paese il sale iodato sia attualmente usato da circa il 70\% delle famiglie e da circa l' $80 \%$ delle mense scolastiche. Inoltre, i dati raccolti mostrano un valore nazionale mediano di ioduria indicativo di iodosufficienza $(125 \mu \mathrm{g} / \mathrm{L})$ e confermano la progressiva scomparsa del gozzo in età scolare nel nostro paese.

La condizione di iodosufficienza in Italia giunge dopo 15 anni dall' approvazione della Legge 55/2005 che, di fatto, ha introdotto il programma nazionale di iodoprofilassi su base volontaria nel nostro paese. Un tempo che può apparire troppo lungo, ma che si giustifica con le scarse risorse che si sono rese disponibili in questi anni per sostenere i costi di promozione, monitoraggio e formazione necessari per l'implementazione di un programma di prevenzione su scala nazionale. A tale difficoltà si è cercato di ovviare con una coordinata ed efficace sinergia di azioni che ha visto istituzioni, società scientifiche, medici di medicina generale, medici specialisti, pediatri di libera scelta, medici dei Servizi di Prevenzione delle Aziende Sanitarie Locali e associazioni dei pazienti lavorare insieme per il raggiungimento di un obiettivo comune: garantire un'adeguata nutrizione iodica alla popolazione italiana. 


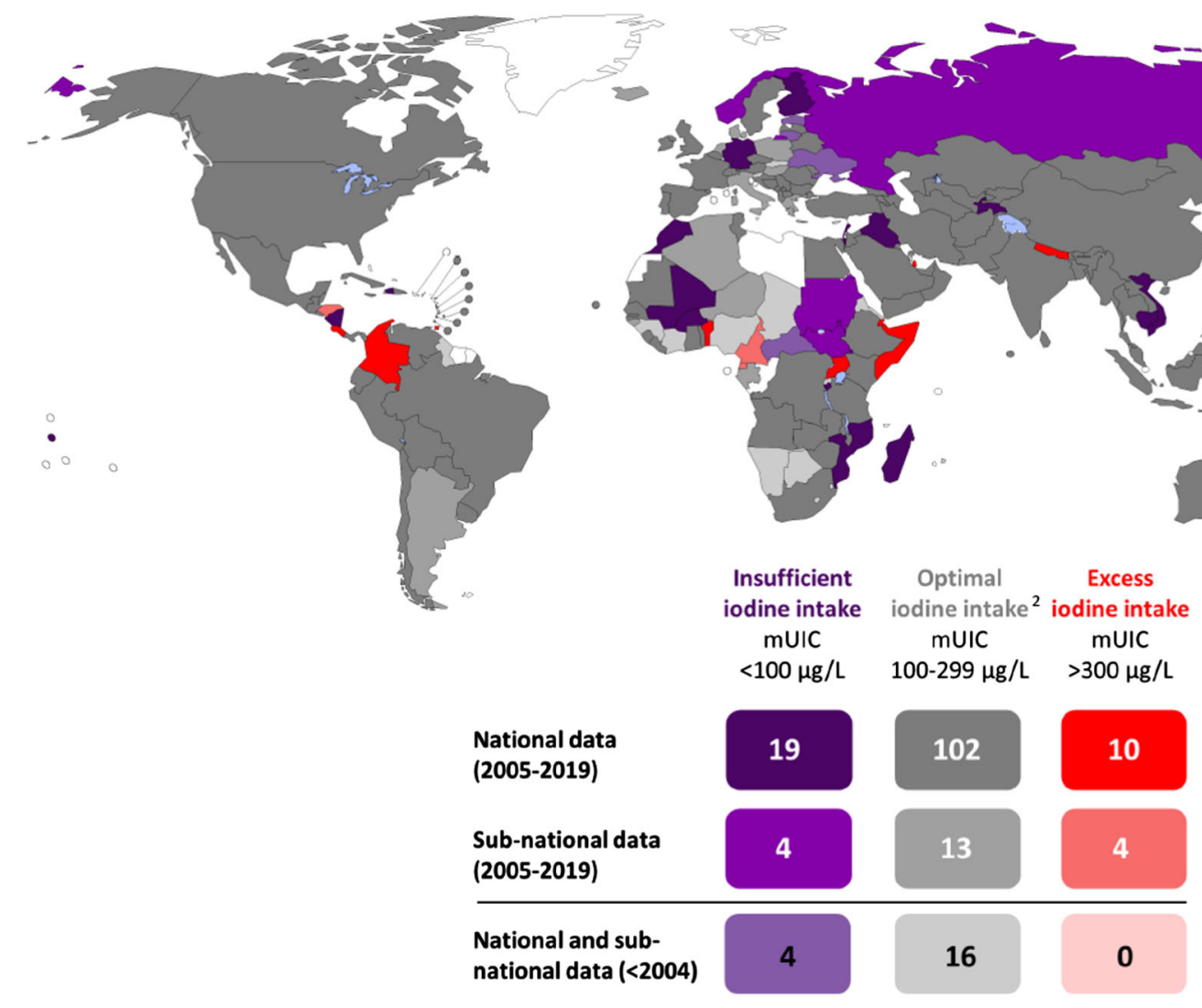

(C) The lodine Global Network, 2020

Fig. 1 Mappa globale della nutrizione iodica basata sulla concentrazione iodica urinaria mediana (mUIC) in bambini in età scolare. La mappa e i dati relativi alla popolazione generale sono riprodotti con autorizzazione Creative Commons CC-BY-SA-ND-NC

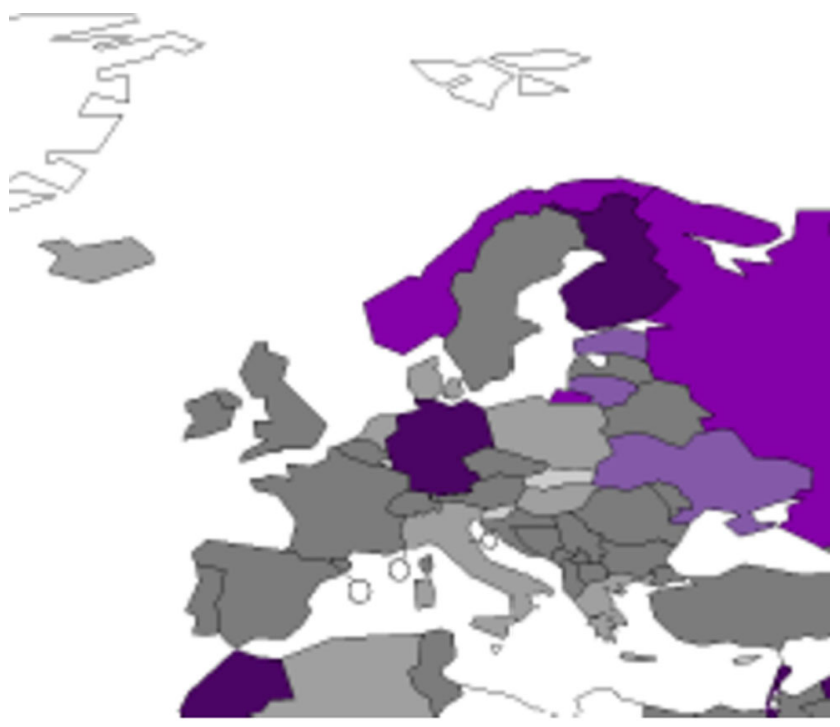

(C) The lodine Global Network, 2020

Fig. 2 Particolare della mappa che indica come ottimale la situazione in Italia
Tale risultato, quindi, rappresenta un traguardo importante per la salute pubblica che assume particolare rilevanza sia per il contesto in cui si è realizzato sia perché la prevenzione dei disordini tiroidei, primo fra tutti il gozzo e le sue complicanze nodulari, consentirà di ottenere un consistente risparmio per il paese in termini di costi sociosanitari. Inoltre, un'adeguata nutrizione iodica fin dalle prime fasi della vita sarà garanzia di un adeguato sviluppo neurocognitivo per i nuovi nati con considerevoli conseguenti vantaggi, anche alla luce del fatto che è stato dimostrato che il prodotto interno lordo di un paese è direttamente proporzionale al QI medio della sua popolazione $[6,7]$.

L'obiettivo che il paese ora dovrà porsi sarà la sostenibilità del programma di iodoprofilassi. Questo potrà essere raggiunto solo attraverso: 1) una contestuale azione di sostegno al programma di riduzione del consumo di sale e al programma di iodoprofilassi attraverso la promozione dello slogan "poco sale ma iodato"; 2) un'efficace azione di monitoraggio che coinvolga anche segmenti di popolazione a maggior rischio di iodocarenza, come le donne in gravidanza per le quali non è stata ancora dimostrata in via definitiva un'adeguata nutrizione iodica nel nostro paese; 3) un'incisiva azione di formazione sull'importan- 
te tema della prevenzione dei disordini da carenza iodica che sia rivolta non solo alle nuove generazioni di medici, nutrizionisti e dietisti, ma anche agli studenti della scuola primaria e secondaria di primo e secondo grado, così come già sperimentato con successo con il protocollo d'intesa tra Istituto Superiore di Sanità, società scientifiche, associazioni dei pazienti e MIUR nel triennio 2016-2019 (http://old.iss.it/binary/osna/cont/Protocollo_MIUR_2016_ 2019.pdf).

Certamente l'approvazione della Legge 55/2005 ha decretato l'impegno politico del nostro paese a contrastare gli effetti gravi per la salute dell'esposizione alla carenza nutrizionale di iodio. Riteniamo che sia giunto il momento che questo impegno venga rinnovato per garantire continuità e sostenibilità al programma nazionale di iodoprofilassi e che, seppure nell' attuale emergenza Covid-19, l'attenzione non venga distolta da un programma di prevenzione del quale solo ora iniziamo ad apprezzare i benefici [8].

Conflitto di interesse Gli autori Antonella Olivieri, Paolo Vitti, Francesco Giorgino e Francesco Trimarchi dichiarano di non avere conflitti di interesse.

Consenso informato Lo studio presentato in questo articolo non ha richiesto sperimentazione umana.

Studi sugli animali Gli autori di questo articolo non hanno eseguito studi sugli animali.
Nota della casa editrice Springer Nature rimane neutrale in riguardo alle rivendicazioni giurisdizionali nelle mappe pubblicate e nelle affiliazioni istituzionali.

\section{Bibliografia}

1. The Iodine Global Network (2020) Global scorecard of iodine nutrition in 2020 in the general population based on school-age children (SAC). IGN, Ottawa, Canada, 2020. https://www.ign. org/cm_data/Global-Scorecard-2020-3-June-2020.pdf. Accessed 26 August 2020

2. Vigneri R (1988) Studies on goiter endemia in Sicily. J Endocrinol Invest 11:831-843

3. Trimarchi F, Ghigo E, Vitti P (2014) I disordini da carenza nutrizionale di iodio in Italia: storia, stato attuale e prospettive di eradicazione. Endocrinologo 15:225-233

4. Olivieri A, De Angelis S, Rotondi D et al (2019) Attività di monitoraggio del programma nazionale per la prevenzione dei disordini da carenza iodica: la situazione italiana a 14 anni dall'approvazione della Legge 55/2005. Endocrinologo 20:245-248

5. Olivieri A, Andò S, Bagnasco M et al (2019) The iodine nutritional status in the Italian population: data from the Italian National Observatory for Monitoring Iodine Prophylaxis-OSNAMI (period 2015-2019). Am J Clin Nutr 110:1265-1266

6. Jones G, Schneider WJ (2006) Intelligence, human capital and economic growth: a Bayesian averaging of classical estimates (BACE) approach. J Econ Growth 11:71-93

7. Jones C, Klenow PJ (2016) Beyond GDP? Welfare across countries and time. Am Econ Rev 106:2426-2457

8. Olivieri A, Trimarchi F, Vitti P (2020) Global iodine nutrition 2020: Italy is an iodine sufficient country. J Endocrinol Invest 43(11):1671-1672 